\title{
Transient Vertical Nystagmus: An Unusual Presentation of Thalamic Infarct
}

Pirasath $\mathbf{S}^{*}$ and Kumanan $\mathbf{T}$

Professorial Medical Unit, Teaching Hospital, Jaffna, Sri Lanka

*Corresponding author: Pirasath S, Professorial Medical Unit, Teaching Hospital, Jaffna, Sri Lanka, Tel: 94775122995; E-mail: selladuraipirasath81@gmail.com

Received date: January 31, 2017; Accepted date: February 24, 2017; Published date: February 28, 2017

Copyright: ( 2017 Pirasath S, et al. This is an open-access article distributed under the terms of the Creative Commons Attribution License, which permits unrestricted use, distribution, and reproduction in any medium, provided the original author and source are credited.

\begin{abstract}
Vertical diplopia and nystagmus typically occur in lesions of the brainstem or cerebellum. The ocular findings are exceedingly rare in thalamic infarction. The vertical gaze paresis is usually involved. Here, we describe a patient presenting with vertical diplopia and nystagmus without gaze paresis secondary to isolated medial thalamic infarct.
\end{abstract}

Keywords: Nystagmus; Diplopia; Thalamus; Infarction

\section{Introduction}

Vertical nystagmus is attributed to a disturbance of the anterior semicircular canal projections and typically indicates a structural lesion of the brainstem. The ocular findings are exceedingly rare in thalamic infarction. Here, we describe a patient presenting with vertical diplopia and nystagmus without gaze paresis secondary to isolated medial thalamic infarct.

\section{Case Report}

A 19-years old previously healthy male presented with a history of vertical diplopia and nystagmus lasting for an hour duration. $\mathrm{He}$ denied vertigo, weakness, paresthesia, or altered level of consciousness. He had no identifiable vascular risk factors or family history of premature cerebrovascular events. On examination, he was oriented with no evidence of language disturbance. Examination of cranial nerves revealed upbeat nystagmus in primary gaze, which intensified on upgaze. Vertical diplopia, was reported in all directions of gaze and was more prominent when focusing on distant objects. There was no evidence of ophthalmoplegia and pupils were symmetrical with intact light reflex. A mild ataxic hemiparesis was evident on presentation. His pulse rate was 80 beats per minute, regular and blood pressure was 120/80 mmHg. Remaining systemic examination was unremarkable. A magnetic resonance imaging (MRI) scan of brain which was performed after the onset of symptoms showed an acute left paramedian thalamic infarct (Figure 1). There were no lesions detected in brainstem or cerebellum. MR angiogram showed intact anterior and posterior circulation. All basic haematological and biochemical investigations including renal and liver profile were normal. Vasculitic and thrombophilic screening were performed and were normal. Cardiac, renal and vascular imaging studies were also normal. Treatment with aspirin and atrovastatin was initiated.

\section{Discussion and Conclusion}

Vertical nystagmus is attributed to a disturbance of the anterior semicircular canal projections and typically indicates a structural lesion of the brainstem at the pontomedullary or pontomesencephalic junctions or cerebellar vermis [1]. Vertical gaze palsy is usually associated with lesions of the mesencephalic rostral interstitial nucleus of the medial longitudinal fasiculus, the interstitial nucleus of Cajal, the posterior commissure and the peri-aqueductal gray matter. Ocular manifestations are exceedingly rare in thalamic infarction and, when present, usually involve vertical gaze paresis [2].
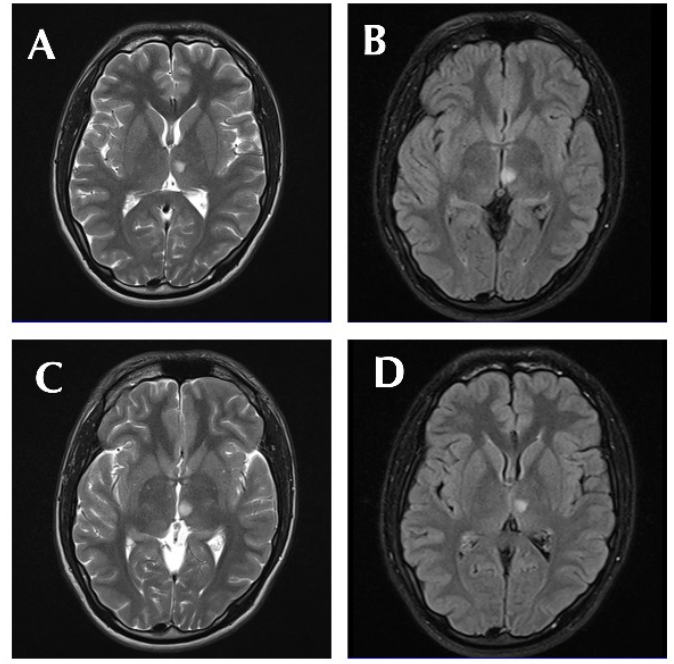

Figure 1: The magnetic resonance imaging (MRI) scan of brain (coronal view) showed an acute left paramedian thalamic infarct.

Thalamus is derived blood supply from vertebrobasilar system via three vessels; namely paramedian thalamo-subthalamic, thalamogeniculate and posterior chorodial arteries and from posterior communicating artery via polar artery [2]. Patient with left thalamic infarction involved in the paramedian thalamo-subthalamic artery territory usually presents altered level of consciousness, contralateral hemiparesis, hemihyperesthesia, diplopia, ptosis, vertical gaze paresis [3]. The medial thalamus is supplied by perforating branches arising from the basilar communicating artery and posterior cerebral arteries [2]. The midbrain is spared because the superior and inferior paramedian mesencephalic arteries arise separately from each other from the basilar communicating artery [2]. 
This patient presented with transient vertical diplopia and nystagmus in the absence of gaze paresis and was found to have a left ventrolateral thalamic infarction on diffuse-weighted magnetic resonance imaging (DWI-MRI). These deficits were likely caused by a transient disturbance in the vestibuloocular pathway transversing the thalamus. Mild ataxic hemiparesis in this patient was explained by involvement of the descending corticobulbar and corticospinal pathways in the adjacent posterior limb of internal capsule [4].

To our knowledge, this is the first case from Sri Lanka describing transient vertical diplopia and nystagmus as manifestations of thalamic infarctions that was detected by DWI-MRI. Findings of vertical diplopia and nystagmus typically suggest an infraction in the midbrain, pons, medulla or cerebellum. However with available advanced technological availability of MRI, these findings can now linked to broader spectrum of anatomical localizations. In our case, transient vertical diplopia and nystagmus as manifestations of the thalamic infarctions that was detected by DWI-MRI. There has been one previous case reported of transient vertical gaze palsy with resolution of symptoms within three hours, highlighting the role of the thalamus in vertical gaze in USA [3]. DWI-MRI has been widely used as imaging modality to detect acute cerebral infarction with sensitivity of $88 \%$ and specificity $95 \%$ [4].

A few previous reports have described vertical gaze palsies in patients with unilateral or bilateral paramedian thalamic infarction, but attributed the gaze palsy to a coexisting midbrain lesion identified primarily at autopsy [5]. The mechanism of vertical gaze paresis with unilateral lesions is uncertain but we can speculate on the possibility of decussation of the frontobulbar fibers in the medial thalamus, as suggested in a case series of thalamic infarctions presenting as vertical gaze palsies [6]. The neuroimaging study results from our patient revealed no midbrain lesion. The absence of gaze paresis was explained by the fact that the infarct extending laterally, thus sparing the mediodorsal nucleus, which sends projections to frontal eye field in the motor cortex [7].

\section{References}

1. Serra A, Leigh RJ (2002) Diagnostic value of nystagmus: Spontaneous and induced ocular oscillations. J Neurol Neurosurg Psychiatry 73: 615-618.

2. Gentilini M, De Renzi E, Crisi G (1987) Bilateral paramedian thalamic artery infarcts: Report of eight cases. J Neurol Neurosurg Psychiatry 50: 900-909.

3. Blitshteyn S, Hentschel K, Czervionke LF, Eidelman BH (2006) Transient vertical diplopia and nystagmus associated with acute thalamic infarction. Clin Imaging 30: 54-56.

4. Küker W, Weise J, Krapf H, Schmidt F, Friese S, et al. (2002) MRI characteristics of acute and subacute brainstem and thalamic infarctions: Value of T2- and diffusion-weighted sequences. J Neurol 249: 33-42.

5. Van der Graaff MM, Vanneste JA, Davies GA (2000) Unilateral thalamic infarction and vertical gaze palsy: cause or coincidence? J Neuroophthalmol 20: 127-129.

6. Clark JM, Albers GW (1995) Vertical gaze palsies from medial thalamic infarctions without midbrain involvement. Stroke 26: 1467-1470.

7. Deleu D (1997) Selective vertical saccadic palsy from unilateral medial thalamic infarction:clinical,neurophysiological and MRI correlates. Acta Neurology Scand 96: 332-336. 